\title{
Revisión de los tumores epidermoides de pene
}

\author{
Diz Rodríguez R, Vírseda Chamorro M, Arance Gil I, Quijano Barroso P, Martínez Benito MªM, \\ Paños Lozano P.
}

Servicio de Urología. Hospital Central de la Defensa. Madrid.

Actas Urol Esp. 2007;31(1):7-10

\section{RESUMEN}

REVISIÓN DE LOS TUMORES EPIDERMOIDES DE PENE

Objetivos. Estudiar las características y evolución de los tumores epidermoides de pene.

Material y métodos. Se realizó un estudio retrospectivo sobre los tumores epidermoides de pene tratados en nuestro centro entre 1981 y 2005.

Resultados. Se diagnosticaron 16 tumores epidermoides de pene. La edad media de los pacientes fue de 71,7 años (intervalo entre 54 y 90 años). En el 80\% de los casos se diagnostican en estadios avanzados (T3 y T4). Las formas de presentación más habituales son las lesiones ulcerosas (53\%) y papilares (33\%), siendo el diámetro medio de la lesión de $2,3 \mathrm{~cm}$, y la localización más frecuente el glande (53\%) y el surco balano prepucial (33\%). Se realizaron 7 escisiones locales, 8 penectomías parciales, una penectomía total, y una escisión más tratamiento local con 5-fluoracilo. Con una media de seguimiento de 24 meses se produjeron 5 recidivas, principalmente en pacientes sometidos a escisión local de la lesión ( $\mathrm{p}=0,06$ test de log-rank).

Conclusiones. El carcinoma epidermoide de pene es una lesión tumoral propia de edades avanzadas que se diagnostica tardiamente y se trata de manera poco agresiva, por lo que son frecuentes los estadios avanzados y las recidivas.

Palabras clave: Carcinoma epidermoide de pene. Tratamiento conservador. Tratamiento quirúrgico

\section{ABSTRACT \\ PENIS EPIDERMOID TUMORS REVIEW}

Objetives. To study the characteristics and evolution of the epidermoid penis tumours.

Material and methods. It was carried out a retrospective study on the epidermoid penis tumours treated in our center between 1981 and 2005.

Results. 16 tumours penis epidermoides were diagnosed. The average age of the patients was of 71,7 years (interval between 54 and 90 years). In $80 \%$ of the cases they are diagnosed in advanced stadiums (T3 and T4). The most habitual presentation forms are the ulcerous lesions (53\%) and papilar (33\%). The average diameter of the lesion was $2,3 \mathrm{~cm}$, and the most frequent localization the glands (53\%) and balano prepucial (33\%). They were carried out 7 local scissions, 8 partial penectomies, onetotal penectomy, and an local scission pluslocal treatment with 5-fluoracile. With a followup of 24 months 5 relapses took place, mainly in patients subjected to local excision of the lesion ( $\mathrm{p}=0,06$ log-rank test).

Conclusions. The epidermoid carcinoma of the penis is a tumoral lesion characteristic of advanced ages that is diagnosed later and treated in not very aggressive way. Therefore it is frequent the advanced stadiums and the tumour relapses.

Keywords: Epidermoid Carcinoma of penis. Conservative treatment. Surgical treatment. 
$\mathrm{E}$ carcinoma epidermoide de pene es un tumor poco frecuente en los países desarrollados. Su incidencia se estima en 1-7,9/100.000 varones $^{1}$.

Debido a su baja incidencia, existen muchas controversias respecto al tratamiento. Algunos autores propugnan un tratamiento conservador para preservar la función sexual ${ }^{2,3}$, mientras que otros consideran indicado un tratamiento más agresivo $^{1}$.

El objetivo de nuestro trabajo es realizar una revisión sobre las principales características de este tipo de neoplasia y la influencia del tratamiento en su evolución.

\section{MATERIAL Y MÉTODOS}

Se realizó un estudio longitudinal retrospectivo, de 16 pacientes tratados de carcinoma epidermoide de pene en nuestro servicio entre enero de 1981 y enero de 2004.

A partir de las historias clínicas de los pacientes se recogieron los datos referentes a su edad, las características macroscópicas de la lesión, tiempo de evolución desde su inicio, diagnóstico de extensión, tratamiento y evolución posterior. El diagnóstico de carcinoma epidermoide se realizó por el servicio anatomía patológica de nuestro centro, previa extirpación de la lesión. El diagnóstico de extensión se realizo mediante la palpación inguinal en busca de adenopatías, y la realización de radiografía de tórax, y TAC abdomino-pélvico ${ }^{4}$. El tiempo de seguimiento medio de los pacientes fue de 24 meses (mediana de 11 meses).

Para comparar la evolución tumoral se utilizó el análisis actuarial de supervivencia con la prueba de log-rank.

\section{RESULTADOS}

La edad media de los pacientes fue de 71,7 años (desviación típica: 10,2 años, intervalo entre 54 y 90 años). El tiempo medio de evolución de la lesión antes de ser diagnosticada fue de 18 meses (mediana de 8 meses).

Las características macroscópicas de las lesiones se muestran en la Tabla 1. Las formas de presentación más comunes fueron la lesión ulcerosa (53\%) y la papilar o verrucosa (33\%). La localización más frecuente fue en glande
(53\%) y surco balano-prepucial (33\%). Su diámetro medio fue de $2,3 \mathrm{~cm}$ (desviación típica $1,4 \mathrm{~cm})$.

El grado de invasión tumoral en el momento del diagnóstico se muestra en la Tabla 2. Un 80\% de los pacientes fueron diagnosticados con un grado de invasión tumoral de T3 y T4. En 4 casos de tumores con grado de invasión T4 se diagnosticaron adenopatías tumorales y en dos casos cursaron con metástasis a distancia.

\section{Tabla 1}

Características macroscópicas de la lesión

\begin{tabular}{lcc}
\hline & $\begin{array}{c}\text { Frecuencia } \\
\text { absoluta }\end{array}$ & $\begin{array}{c}\text { Porcentaje } \\
\text { registrados }\end{array}$ \\
\hline $\begin{array}{l}\text { Forma de presentación } \\
\text { Papilar }\end{array}$ & 5 & $33 \%$ \\
Nodular & 1 & $7 \%$ \\
Ulcerosa & 8 & $53 \%$ \\
Plana & 1 & $7 \%$ \\
No registrada & 1 & - \\
Total & $\mathbf{1 6}$ & $\mathbf{1 0 0} \%$ \\
Localización & & \\
Prepucio & 1 & $7 \%$ \\
Glande & 8 & $53 \%$ \\
Surco & 5 & $33 \%$ \\
Dorso & 1 & $7 \%$ \\
No registrado & 1 & - \\
Total & $\mathbf{1 6}$ & $\mathbf{1 0 0 \%}$ \\
Número de lesiones & & $\mathbf{1 0 0 \%}$ \\
Única & 12 & $80 \%$ \\
Múltiple & 3 & $20 \%$ \\
No registrado & 1 & - \\
Total & & \\
\hline
\end{tabular}

Tabla 2

Invasión tumoral en el momento del diagnóstico

\begin{tabular}{lcc}
\hline & $\begin{array}{c}\text { Frecuencia } \\
\text { absoluta }\end{array}$ & $\begin{array}{c}\text { Porcentaje } \\
\text { registrados }\end{array}$ \\
\hline $\mathrm{T} 1$ & 1 & $7 \%$ \\
$\mathrm{~T} 2$ & 2 & $13 \%$ \\
$\mathrm{~T} 3$ & 5 & $33 \%$ \\
$\mathrm{~T} 4$ & 7 & $47 \%$ \\
No registrado & 1 & - \\
Total & $\mathbf{1 6}$ & $\mathbf{1 0 0} \%$ \\
\hline
\end{tabular}


Se realizaron 7 escisiones locales, 8 penectomías parciales, una penectomía total, y una escisión local más tratamiento local con 5-fluoracilo. En dos casos de tumores T4 el tratamiento se completó con linfadenectomía inguinal bilateral.

La evolución de la neoplasia según el diámetro tumoral, grado tumoral, tipo de crecimiento, afectación linfática se muestra en la Tabla 3, la relación entre estadio tumoral y tipo de tratamiento se muestra en la Tabla 4. La influencia del estadio tumoral y de la linfadenectomía en las recidivas tumorales se muestra en la Tabla 5. El número de recidivas tumorales registradas durante el seguimiento fue de cinco. El principal factor que influyó en la aparición de recidiva tumoral fue el tipo de tratamiento. Las recidivas fueron más frecuentes en pacientes tratados con escisión local de la lesión, frente a los tratados con amputación peneana ( $\mathrm{p}=0,06$ prueba de log-rank) (Fig. 1).

Tabla 3

Relación factores pronósticos/recidiva neoplásica

\begin{tabular}{lllll}
\hline Factor pronóstico & \multicolumn{2}{c}{ Recidiva } & Significación \\
& & \multicolumn{1}{c}{ No } & Si & \\
\hline Diámetro tumoral $(*)$ & & $2,19(1,13)$ & $2,40(1,91)$ & $0,829(\dagger)$ \\
Grado tumoral & G1 & $3(50 \%)$ & $3(50 \%)$ & $0,326(\dagger)$ \\
& G2 & $3(60 \%)$ & $2(40 \%)$ & \\
& G3 & $3(100 \%)$ & 0 & \\
Tipo de crecimiento & Superficial & $2(50 \%)$ & $2(50 \%)$ & $0,631(\dagger)$ \\
& Vertical & $2(100 \%)$ & 0 & \\
& Verrucoso & $3(60 \%)$ & $2(40 \%)$ & \\
& Profundo & $3(75 \%)$ & $1(25 \%)$ & \\
& No & $6(60 \%)$ & $4(40 \%)$ & $0,597(\dagger)$ \\
Estadio linfático & $>$ No & $3(75 \%)$ & $1(25 \%)$ & \\
& & &
\end{tabular}

*Media (entre paréntesis desviación típica) en $\mathrm{cm}$. † No significativo.

Tabla 4

Evolución tumoral según la invasión tumoral y tipo de tratamiento

\begin{tabular}{lccccc}
\hline $\begin{array}{l}\text { Estadio } \\
\text { tumoral }\end{array}$ & $\begin{array}{c}\text { Escision } \\
\text { local }\end{array}$ & $\begin{array}{c}\text { Penectomia } \\
\text { parcial }\end{array}$ & $\begin{array}{c}\text { Tratamiento } \\
\text { Penectomia } \\
\text { total }\end{array}$ & $\begin{array}{c}\text { Escisión }+ \\
\text { 5-fluoracilo }\end{array}$ & Total \\
\hline $\mathrm{T} 1$ & $1(1)$ & 0 & 0 & 0 & $1(1)$ \\
$\mathrm{T} 2$ & $2(1)$ & 0 & 0 & 0 & $2(1)$ \\
$\mathrm{T} 3$ & $3(1)$ & $2(0)$ & & $1(1)$ & $5(2)$ \\
$\mathrm{T} 4$ & 0 & $6^{*}(1)$ & $1^{*}$ & & $7(1)$ \\
Perdido & 1 & - & - & - & 1 \\
\hline
\end{tabular}

Entre paréntesis número de pacientes que presentaron recidiva. (*) El tratamiento se completó con linfadenectomía bilateral.

\section{DISCUSIÓN}

El carcinoma epidermoide es un tumor que afecta principalmente a adultos de edad avanzada. La edad media de nuestra serie fue de 71,7 años. En otras series su edad media oscilo ente $\operatorname{los} 54^{5}$ y los 66 años ${ }^{6}$. Parece ser que en los pacientes de raza negra la aparición de este tipo de tumor es más precoz ${ }^{7}$.

El tipo característico de presentación es la lesión ulcerada, seguida de la infiltrante/ profunda y la papilar o verrucosa ${ }^{8}$. En nuestra serie se observó un gran porcentaje de pacientes con lesión de tipo verrucoso, quizás en relación con una posible infección por el virus del papiloma humano ${ }^{9}$. Respecto a su localización otras series encuentran un mayor porcentaje de localizaciones en prepucio seguida del glande, mientras en nuestro caos fue más frecuente en glande y surco balano-prepucial. Estas diferencias pueden guardar relación con los factores de riesgo del carcinoma peneano ${ }^{10}$. La fimosis sería un importante factor de riesgo, mientras que en nuestra serie parece por el tipo de lesión, que sería más frecuente como factor de riesgo la infección por el virus del papiloma humano. Los pacientes de nuestra serie presentados estadios tumorales avanzados en el momento del diagnóstico, probablemente relacionado con el tiempo transcurrido desde la aparición de la lesión hasta su diagnóstico (19 meses de media). Otras series refieren que la mayoría de los tumores son diagnosticados en un estadio inicial o superficial $^{6,11}$. Aunque no son infrecuentes los diagnósticos en etapas más tardías ${ }^{5,10}$.

Existe controversia respecto al tratamiento. Algunos autores proponen tratamientos conservadores con láser para los tumores peneanos localizados, con el fin de no alterar la función sexual de estos pacientes ${ }^{12}$. Sin 
Tabla 5

Influencia del estadio tumoral y la linfadenectomía en la aparición de recidivas tumorales

\begin{tabular}{lllll}
\hline Factor pronóstico & \multicolumn{2}{c}{ No } & \multicolumn{1}{c}{ Si } & Significación \\
& & 0 & $1(100 \%)$ & $0,322(\dagger)$ \\
\hline Estadio tumoral & $\mathrm{T} 1$ & $1(50 \%)$ & $1(50 \%)$ & \\
& $\mathrm{T} 2$ & $3(60 \%)$ & $2(40 \%)$ & \\
& $\mathrm{T} 3$ & $6(86 \%)$ & $1(14 \%)$ & \\
& $\mathrm{T} 4$ & $9(64 \%)$ & $5(36 \%)$ & $0,308(\dagger)$ \\
Linfadenectomía & $\mathrm{No}$ & $2(100 \%)$ & 0 & \\
& $\mathrm{Si}$ & & & \\
\hline
\end{tabular}

El factor pronóstico del grado de infiltración tumoral también es motivo de controversia. Algunos autores niegan que exista relación entre este factor y las tasas de recidivas o intervalo libre de enfermedad $^{1,17}$, mientras que para otros autores consideran a este factor junto con la afectación linfática como factores pronósticos principales ${ }^{11}$. En nuestro estudio, aunque con un limitado número de casos, observamos

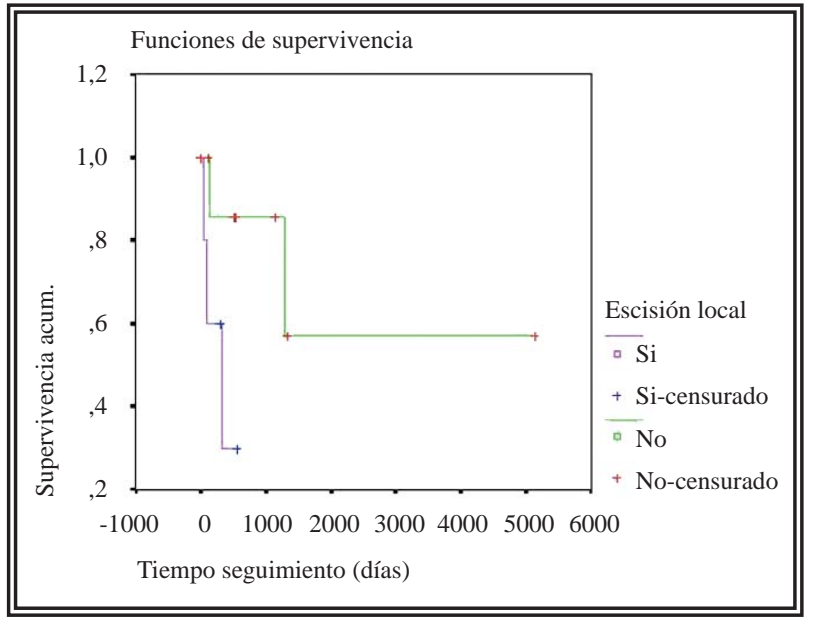

FIGURA 1. Función de supervivencia según el tipo de tratamiento.

embargo, otros autores proponen la Penectomía parcial incluso para lesiones superficiales ${ }^{6}$, con unas tasas de recidivas del 11\%. Otros autores con tratamiento conservador local (braquiterapia más escisión local) refieren una tas de recidivas del $39 \%^{13}$, similares a las nuestras. Recientemente se ha realizar linfadenectomía precoz en estadios donde clínicamente no se observan adenopatías inguinales ${ }^{14}$. En nuestro trabajo ninguno de los pacientes a los que se les realizó linfadenectomía experimentaron recidiva, pero debido al pequeño número de casos no podemos extraer conclusiones estadísticas.

Entre los factores pronósticos que intervienen en la supervivencia del tumor de pene se encuentran la palpación de adenopatías inguinales, el grado histológico y el estadio patológico linfático $(\mathrm{N})^{15}$. Aunque los análisis multivariantes parecen indicar que es el estadio patológico tumoral el principal factor pronóstico ${ }^{16}$. que el tipo de tratamiento fue el factor que más influyó en la evolución de este tipo de tumores.

\section{REFERENCIAS}

1. Mobilio G. Ficarra V. Genital treatment of penile carcinoma. Curr Opin Urol. 2001;11(3):299-304.

2. Hakenberg OW, Wirth MP Issues in the treatment of penile carcinoma. A short review. Urol Int. 1999;62(4):229-233.

3. Ficarra V, Maffei N, Piacentine I, Al rabi N, Cerruto MA, Artibani W. Local treatment of penile squamous cell carcinoma. Urol Int. 2002;69(3):169-173.

4. Sobin LH, Wittekind Ch.TMN clasification of malignan tumors. $6^{\text {th }}$ edition. New York: Wiley-Liss, 2002.

5. Heyns CF, van Vollenhoven P, Steenkamp JW, Allen FJ. Cancer of the penis-a review of 50 patients. S Afr J Surg. 1997;35(3):120-124

6. Banon Perez VJ, Nicolas Torralba JA, Valdelvira Nadal P, Server Pastor G, Garcia Hernandez JA, Guardiola Mas A et al. Carcinoma escamoso de pene. Arch Esp Urol. 2000;53(8):693-699.

7. Rippentrop JM, Joslyn SA, Konety BR. Squamous cell carcinoma of the penis: evaluation of data from the surveillance, epidemiology, and end results program. Cancer. 2004;15;101(6):1357-1363.

8. Villavicencio H, Rubio-Briones J, Regalado R, Chechile G, Algaba F, Palou J. Grade, local stage and growth pattern as prognostic factors in carcinoma of the penis Eur Urol. 1997;32(4):442-447.

9. Maiche AG Epidemiological aspects of cancer of the penis in Finland. Eur J Cancer Prev. 1992;1(2):153-158.

10. Hunter-Mellado R, Rodriguez P. Squamous cell carcinoma of the penis. Bol Asoc Med P R. 1990;82(9):416-418.

11. Soria JC, Fizazi K, Piron D, Kramar A, Gerbaulet A, Haie-Meder C. Squamous cell carcinoma of the penis: multivariate analysis of prognostic factors and natural history in monocentric study with a conservative policy. Ann Oncol 1997;8(11):1089-1098.

12. Windahl T, Skeppner E, Andersson SO, Fugl-Meyer KS. Sexual function and satisfaction in men after laser treatment for penile carcinoma. $\mathrm{J}$ Urol. 2004;172(2):648-651.

13. Kroon BK, Horenblas S, Lont AP, Tanis PJ, Gallee MP, Nieweg OE. Patients with penile carcinoma benefit from immediate resection of clinically occult lymph node metastases. J Urol. 2005;173(3):816-819.

14. Leewansangtong S, Srinualnad S, Chaiyaprasithi B, Taweemonkongsap T, SoontrapaS. The risks of lymph node metastasis and the prognostic factors in carcinoma ofthe penis: analysis of 50 patients treated with bilateral ilioinguinal lymphadenectomy. J Med Assoc Thai. 2001;84(2):204-211.

15. Pandey D, Mahajan V, Kannan RR. Prognostic factors in node-positive carcinoma of the penis. J Surg Oncol. 2006; 1;93(2):133-138.

16. Lopes A, Hidalgo GS, Kowalski LP, Torloni H, Rossi BM, Fonseca FP. Prognostic factors in carcinoma of the penis: multivariate analysis of 145 patients treated with amputation and lymphadenectomy. J Urol. 1996;156(5):1637-1642.

17. Rozan R, Albuisson E, Giraud B, Boiteux JP, Dauplat J. Epithelioma of the penis treated with surgery. Study Group on Urogenital Tumors of the National Federation of the Centers for Cancer Control. Prog Urol. 1996;6(6):926-935.

Correspondencia autor: Dr. M. Virseda Chamorro. Servicio de Urología. Hospital Central de la Defensa. Plza. del Ejército, s/n. Ciudad Universitaria. 28040 Madrid. Tel.: 914520400

E-mail autor: bgmeli@terra.es

Información artículo: Revisión - Cáncer de pene

(Trabajo aceptado octubre 2006) 